\title{
Sealants effective in reducing caries
}

\author{
Lodra J C, Bravo M, Delgado-Rodriguez M, Baca P, Galvez R. Factors influencing the effectiveness of sealants - a \\ meta-analysis. Community Dent Oral Epidemiol 1993; 21: 261-268
}

Objective To determine the effectiveness of fissure sealants and factors influencing their effectiveness.

Data sources Medline search 1975-1990 (pit and fissure sealants), hand search of Medline references identified and 11 relevant dental journals no unpublished studies were sought.

Study selection Studies were included if they contained original data, were applied to original data, no other preventive measure was used, the effectiveness of sealants could be assessed. Studies in English, French of Spanish were included. All articles were evaluated by two authors to minimise selection and data bias. Where several articles reported the same study only data from the most recent was used.

Data extraction and synthesis The prevented fraction was calculated for each study. Heterogeneity between the studies was investigated using multivariate analysis. Data was analysed for both auto-polymerising and light-cured(UV) sealants.

Results 24 studies meeting the criteria were identified in 34 articles. Only UV light cured and auto-polymerising sealant studies met the criteria so no non UV light-cured were included. The percentage of caries prevented (prevented fraction) is shown in the table. The auto-polymerising sealants were clearly more effective. Effectiveness was shown to decrease with time for both types.
Effectiveness may be improved in fluoridated communities $(83 \%$ c.f. $71 \%$ ) and operator type may also influence effectiveness.

\begin{tabular}{lcc}
\hline Sealant type & Prevented fraction (\%) & $95 \% \mathrm{CI}$ \\
Auto & 71.36 & $69.69-72.94$ \\
Light (UV) & 45.92 & $43.5-48.24$ \\
\hline
\end{tabular}

There was a marked heterogeneity between the studies which was partly explained by fluoridation status and incidence of caries in the control group. The authors also note that publication bias ( the tendency for reporting positive studies over negative or non-significant findings) could have been a factor.

Conclusions Fissure sealants are effective in preventing dental caries, their effectiveness decreases with time, and periodic reapplication is advisable. Auto-polymerising sealants are more effective than UV light sealants. Fluoridation appears to increase effectiveness, so future studies should note fluoride consumed by study population. Further studies should look at the influence of the operator on effectiveness.

Address J C Lodra, Department of Preventive Dentistry, School of Dentistry, University of Granada, Spain

Funding University of Granada Grant no. PG/89-24199739

\section{Commentary}

The evidence that sealants are effective in both preventing caries and arresting the progress of caries is overwhelming. This article compares auto-polymerised light-cured resins available before 1990 . They arrive at the conclusion that autopolymerising resins provide more benefit than light-cured resins (NNT = 4). This report, however, does not address several important questions.

The first issue is evolution. Technically speaking, the current (1998) generation of light-cured, floatable composite resins and the lights used to cure them, are several generations removed from those reported by Lodra. The current sealants and the floatable resins provide significant improvements in low viscosity composite technology, with better strength, seals and wear resistance.

The second issue is clinical experience. The current generation of products is more clinically controllable than those of previous eras. They are easier to use and take less clinical time.

The third issue asks the question: 'Are 16 ounces of prevention worth a pound of cure?. In a fluoridated community with a low incidence of community and patient caries and a fee-for-service basis, the cost of repeated application of sealants can far exceed the cost of restoring an occasional occlusal carious lesion - finances favour no sealants. On the other hand, given the same situation in a non-fluoridated area with a high caries incidence, the financial considerations are reversed - the finances favour sealants. More complex is a financial analysis of capitated or prevention environment. The economics depend on additional variables, including risk assessment of the patient, risk assessment of the teeth, capitation fees, salaries, time and materials.

The fourth issue is how might an ideal comprehensive dental care practice with a preventive mission use this information. All available evidence indicates that sealants, when appropriately applied, are an extraordinarily effective preventive procedure for both children and adults (and this includes sealing amalgam restorations).

\section{Michael Ferguson}

Professor of Restorative Dentistry, School of Dental Medicine, Harvard University, Boston, USA 